\title{
The Observatorio Astronómico Nacional - Tonantzintla: Site Evaluation
}

\author{
H.M. Hernández-Toledo ${ }^{1}$, L.A. Martínez-Vázquez ${ }^{1}$ and A. Pani-Cielo ${ }^{2}$ \\ ${ }^{1}$ Instituto de Astronomía, Universidad Nacional Autónoma de México, \\ Ciudad Universitaria. A.P. 70-264, C.P. 04510, México D.F., México \\ email: hector@astroscu.unam.mx \\ ${ }^{2}$ Observatorio Astronómico Nacional - Tonantzintla \\ Luis Enrique Erro, Tonantzintla, Puebla 72840, México \\ email: apani@astroscu.unam.mx
}

\begin{abstract}
.
The objective of this contribution is to present some results of an evaluation on the local conditions at the site that were considered in order to propose that the Observatorio Astronómico Nacional, Tonantzintla, (OAN-Tonantzintla) become a National Facility for Astronomy Education. The evaluation included a quantitative diagnostic (CCD photometry) on the quality of the local sky. The attributes of the 1-m telescope, the current instrumentation and a well planned upgrading that includes new instrumentation is considered at the basis for a successful transition maintaining the attractiveness of the site for astronomy education. A 3-year upgrading program actually in progress at UNAM is providing funding for that purpose. Physics and astronomy programs at college and graduated levels at UNAM will benefit from this, yielding clear connections among astronomy researchers and educators and students at various levels. Although the OAN-Tonantzintla faces the danger of deteriorating its sky conditions, we are maintaining awareness of the night sky characteristics in long-term monitoring campaigns and encouraging the local authorities to find alternative solutions to this problem.
\end{abstract}

Keywords. Optical Observatories, Site Evaluation, Education

\section{Introduction}

The Observatorio Astronómico Nacional at Tonantzintla (hereafter OAN-Tonantzintla), under supervision of Instituto de Astronomía at the Universidad Nacional Autónoma de México (IA-UNAM), is located near the Cholula village in the state of Puebla $150 \mathrm{~km}$ away from the Instituto de Astronomía headquarters in México City. Due to the increasing amount of light pollution from the growing city of Puebla and nearby villages, the observatory became progressively unappropriate for deep astronomical observations. Observing priorities changed at Tonantzintla observatory and since the end of decade of the 1970s the site has provided fundamental support for (i) instrumental development and testing (Voitsekhovich et al. 2005) (ii) observing programs of bright objects of various types (Pişmiş et al. 1984) and (iii) public outreach and physics/astronomy university programs.

A close collaboration between Facultad de Ciencias and Instituto de Astronomía at UNAM brought the 1-m telescope of the OAN-Tonantzintla as one of the first professional telescopes in México available at the college level providing physics and astronomy students real access to modern scientific instruments. The acquisition of a scientific CCD at the end of the decade of the 1980s opened new possibilities for both photometric and spectroscopic observations at the site. The 1-m telescope is considered as a highly effective tool for astronomy education and the site itself is an appropriate space that enables 
groups of students to participate easily and safely. In this contribution we briefly present some results of our evaluation of the local sky conditions that were considered to propose the OAN-Tonantzintla as a National Facility for Astronomy Education.

\section{The OAN-Tonantzintla 1-m telescope}

The main telescope at Tonantzintla observatory is a 1-m $f / 15$ Cassegrain with an equatorial mount yielding a plate scale of $13^{\prime \prime} .53 / \mathrm{mm}$ in the focal plane. The range of movements is $\pm 5.6 \mathrm{~h}$ in hour angle and from -60 to $80^{\circ}$ in declination with a maximum pointing velocity of $1^{\circ} / \mathrm{sec}$ and pointing error $\leqslant 1^{\prime}$ for $z \leqslant 60^{\circ}$. The main optical detector is a thinned Metachrome II covered Thomson THX 31156 CCD of 1024 $\times 1024$ pixels, each of $19 \mu \mathrm{m}$ in size. Among its characteristics are a deep well $\sim 175,000$ $e^{-}$, a dark current of $\sim 0.31 e^{-}$hour/pixel at gain mode 4 , a readout noise of $\sim 3.5$ $e^{-}$RMS and a reading rate of $50 \mathrm{KHz}$. The telescope and detector combination attain a maximum field of view of $4.2 \times 4.2$. A set of broad-band $B(\lambda 4300 \AA), V(\lambda 5400 \AA)$, $R(\lambda 6400 \AA), I(\lambda 8900 \AA)$ filters in the Johnson-Cousins systems is available in direct imaging mode. A classic Cassegrain (Boller \& Chivens) spectrograph is also available at the OAN-Tonantzintla. Among its principal components are a set of diffraction gratings of 150, 400, 600 and 830 groove/mm, and a He-Ar comparison lamp. For more details see http://www. astroscu.unam.mx/Tonantzintla.

Figure 1 shows an external view of the telescope building while Figure 2 shows an internal view. The mounting, the main telescope and the cryogenic device that hosts the CCD detector are appreciated.

\subsection{The Sky conditions at OAN-Tonantzintla}

The surface brightness of the moonless night sky is a fundamental quantity of an observing site. The sky brightness at the OAN-Tonantzintla is being degraded with time, as the nearby population center of Puebla and its attendant light pollution has been growing. Sky brightness was measured in our CCD frames at different observing bands at moonless conditions. Since we have observations at different heights above the horizon, the sky brightness at those heights could be estimated as well. Our results are presented in

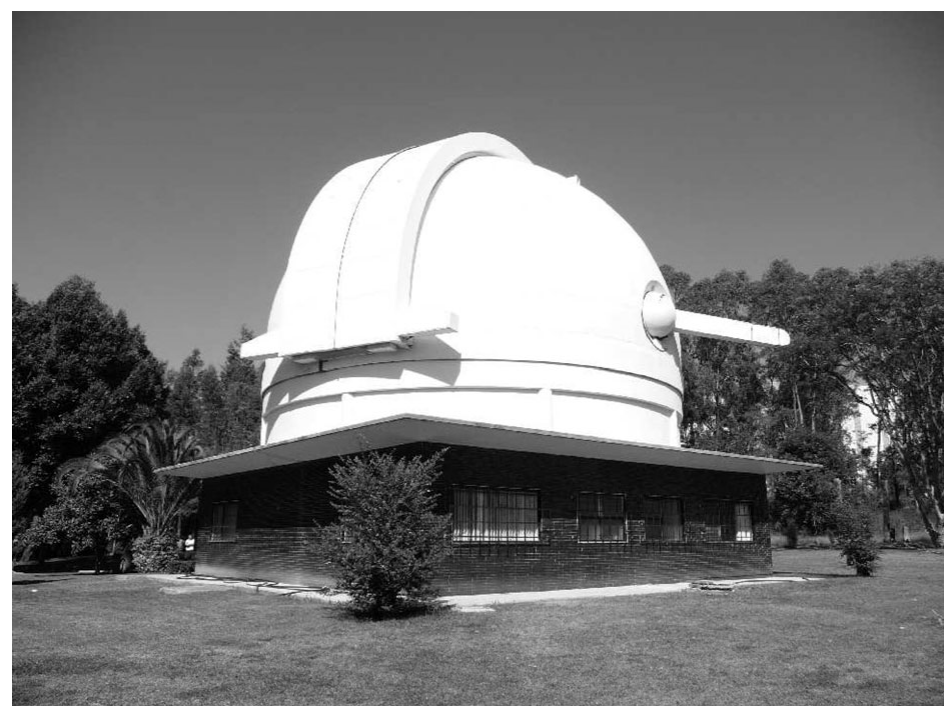

Figure 1. An external view of the 1-m telescope building at the OAN-Tonantzintla. 
Table 1. Airmass and sky surface brightness at OAN-SPM under dark and bright sky conditions. Similar quantities for the OAN-Tonantzintla at two different directions: towards the city of Puebla/local zenith are also presented.

\begin{tabular}{lcccccc}
\hline Band & $X$ (air mass) & Sky & $X$ (air mass) & Sky & $X$ (air mass) & Sky \\
\hline U & 1.00 & 21.3 & 1.09 & 18.9 & & \\
B & 1.00 & 22.3 & 1.12 & 19.5 & $2.1 / 1.05$ & $18.6 / 19.1$ \\
V & 1.01 & 21.4 & 1.15 & 19.4 & $2.2 / 1.09$ & $18.5 / 19.0$ \\
R & 1.00 & 20.9 & 1.18 & 19.3 & $2.3 / 1.12$ & $18.3 / 18.9$ \\
I & 1.02 & 19.4 & 1.21 & 18.2 & $2.4 / 1.16$ & $16.8 / 17.7$ \\
\hline
\end{tabular}

Notes:

OAN-SPM Sky Surface Brightness data from M. Richer (http://www.astrossp.unam.mx/sitio/brillo\_cielo. htm.

Table 1. Column (1) indicates the band of the observation, Column (2) is the airmass at the time of the observation, Column (3) the sky brightness values obtained at the Observatorio Astronómico Nacional San Pedro Mártir (OAN-SPM) under dark sky conditions. Similarly, Columns (4) and (5) represent the a irmass and the corresponding sky brightness values obtained at OAN-SPM under bright (near full-moon) sky conditions. The airmasses at the time of our observations at two different airmasses are presented in Column (6). Finally, the corresponding sky brightness estimates at the OAN-Tonantzintla in dark sky conditions are presented in Column (7).

The results in Table 1 indicate that a representative sky surface brightness value in the $V$ band at the OAN-Tonantzintla is $\sim 18.5 \pm 0.2 \mathrm{mag} \operatorname{arcsec}^{-2}$ to the line of sight to the city of Puebla and $\sim 19.0 \pm 0.2 \mathrm{mag} \operatorname{arcsec}^{-2}$ at the local zenith area. Compared to the representative $V$ band sky value for the darkest site in the northern hemisphere; The Observatorio Astronómico Nacional, San Pedro Mártir at moonless conditions, this difference of about 3 magnitudes is interpreted as the amount of the light pollution contributed to the site from both the local villages around Tonantzintla and from the

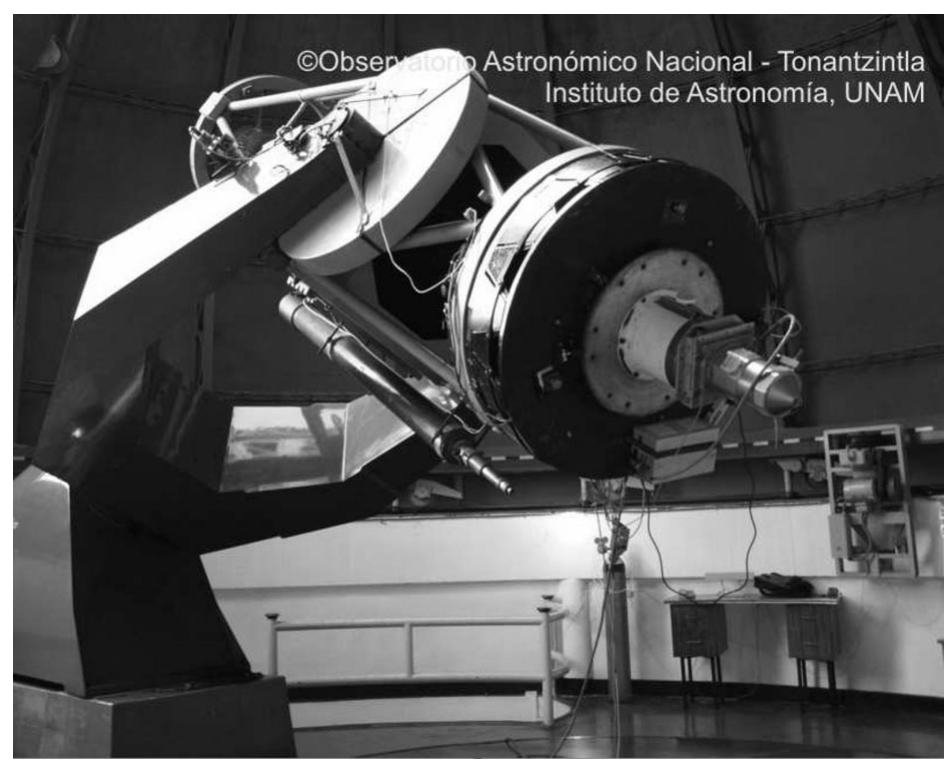

Figure 2. An internal view of the 1-m telescope building at the OAN-Tonantzintla. The mounting, the main telescope and the cryogenic device that hosts the CCD detector are appreciated. 
city of Puebla. The effect is equivalent to loosing the ability to distinguish by eye stars of magnitudes fainter than $\sim 4$ in a clear dark night sky. To complement our sky brightness measures, we carried out a first campaign to estimate the sky surface brightness in a $\sim 8 \mathrm{~km}^{2}$ area around the OAN-Tonantzintla. These results are presented by VázquezMata et al. in this volume (see page ??).

\section{Discussion and conclusions}

The most relevant environmental factor for the OAN-Tonantzintla is lighting. There are two distinct effects. First artificial light scatters in the atmosphere and appears as a background glow to the night sky. This diminishes the contrast of astronomical images, and it is specially injurious to faint objects such as galactic nebulae or external galaxies. Second, outdoor or indoor lighting that is directly visible interferes with the ability of the eye to become fully dark-adapted and thus to see and appreciate the natural night sky including the Milky Way and fainter stars. The former effect is important for professional astronomical research, but it is less important in an educational context. The glow visible at the OAN-Tonantzintla is a combination of contributions from México city and Puebla. The OAN-Tonantzintla thus faces the danger of deteriorating its sky conditions even more. However, to maintain competitiveness for educational and other scientific programs, we are giving the fight to preserve sky brightness conditions through (1) monitoring of the night sky characteristics in various campaigns and (2) encouraging the local authorities about the need to regulate public lighting and at the same time, showing the benefits (economic impact) of such initiatives when well planned and correctly implemented.

The results presented here are part of the requisites that need to be discussed and evaluated to propose that the Observatorio Astronómico Nacional, Tonantzintla, become a National Facility for Astronomy Education. The OAN-Tonantzintla is within relatively easy travel from two major population centers (México city and Puebla) and not so far from other important cities in the central/south part of the country. Most of the infrastructure needed for teachers/student-training workshops and other programs is already in place or in the process of being adapted: visitors/students staying for more than a day can take advantage of appropriate rooms at the observatory campus. Direct contact among teachers, students and active researchers is an important source of inspiration and it is essential for ensuring the quality of programs. Various educational programs at the college level between Instituto de Astronomía and Facultad de Ciencias at UNAM are in a preparative phase with the idea of making connections between researchers and educators. For a more detailed discussion that includes the properties of the local extinction law, a spectroscopic evaluation of the sky, as well as a discussion on various astronomical/educational projects around which a major educational program can be organised, see (Hernandez-Toledo et al. 2010).

\section{References}

Hernández-Toledo, H.M., Martínez-Vázquez, L.A., Moreno-Corral, M.A., Pani-Cielo, A. 2010, Revista Mexicana Astronomía Astrofísica, 46, 47

Pişmiş, P., Hasse, I., Moreno, M.A. 1984, BAAS, 16, 959

Voitsekhovich, V.V., Sánchez, L.J., Orlov, V.G., Garfias, F., Benítez, R. 2005, Revista Mexicana Astronomía Astrofísica, 41, 399 